\title{
SETAC Europe Young Scientist LCA Award 2018 for Rickard Arvidsson
}

\author{
Joost Dewaele ${ }^{1}$ \\ Received: 22 March 2018 / Accepted: 30 March 2018 / Published online: 31 May 2018 \\ (C) Springer-Verlag GmbH Germany, part of Springer Nature 2018
}

At the 28th SETAC Europe annual meeting, 13-17 May 2018, Rome (Italy), Rickard Arvidsson received the SETAC Europe Young Scientist LCA Award 2018.

Rickard isr a bio-engineer by formation (Chalmers University of Technology, Sweden), and finished his $\mathrm{PhD}$ in 2012. His PhD research focused on the development and application of emission and exposure assessment methods for manufactured nanomaterials, thus contributing to the final risk assessment of such materials. More recently, Rickard's research increased focus to life cycle assessment of nanomaterials and other emerging technologies.

The SETAC Europe LCA Steering Committee recognizes Rickard for outstanding contributions to advancing life cycle assessment of emerging technologies, especially in the form of a methodological paper for prospective LCA, which extracts best available empirical evidence gathered over a series of case studies (self-conducted + literature). The resulting definitions and recommendations are considered instrumental in future research and practical application of prospective LCA for emerging technologies, where production scale and future changes in background systems are crucial elements.
Further contributions include the development of a framework towards more consistent reporting and use of energy indicators in LCA, and a novel method for human health impact assessment in social LCA that quantifies the negative as well as the positive product-related health impacts. Through this work, Rickard has demonstrated his ability to identify and solve relevant challenges in life cycle inventory and life cycle impact assessment.

Rickard is the well-deserved winner of the 2018 SETAC Europe Young Scientist LCA Award. With this, we congratulate Rickard with the award, with the work already accomplished, and wish him a fruitful continuation of his activities in his position of Associate professor at Chalmers University of Technology, Sweden.

SETAC Europe Young Scientist LCA Award is sponsored by Lenzing AG (https://www.lenzing.com) and Springer Verlag (http://www.springer.com).

Heinz Stichnothe (Chair)/Joost Dewaele (Award Manager) on behalf of the SETAC Europe LCA steering committee

Joost Dewaele

dewaele.j@pg.com

1 Procter and Gamble, Strombeek-Bever, Belgium 